\title{
Asteroseismology and Forced Oscillations of the Simultaneous p-Mode and g-Mode Pulsator HD 209295
}

\author{
G. Handler, ${ }^{1}$ L. A. Balona,${ }^{1}$ R. R. Shobbrook,${ }^{2,3}$ C. Koen, ${ }^{1}$ A. Bruch, ${ }^{4}$ \\ E. Romero-Colmenero, ${ }^{1}$ A. A. Pamyatnykh,${ }^{5}$ B. Willems, ${ }^{6}$ L. Eyer,${ }^{7,8}$ \\ D. J. James, ${ }^{9,10,11,12}$ T. Maas, ${ }^{7}$ L. A. Crause ${ }^{13}$
}

${ }^{1}$ South African Astronomical Observatory, P.O. Box 9, Observatory 7935, South Africa; ${ }^{2}$ P.O. Box 518, Coonabarabran, N.S.W 2357, Australia; ${ }^{3}$ Research School of Astronomy and Astrophysics, Australian National University, Weston Creek P.O., ACT 2611, Australia; ${ }^{4}$ Labóratorio Nacional de Astrofísica, Itajubá, Brazil; ${ }^{5}$ Copernicus Astronomical Center, ul. Bartycka 18, 00-716 Warsaw, Poland; ${ }^{6}$ Department of Physics and Astronomy, The Open University, Walton Hall, Milton Keynes $M K^{7}$ 6AA, UK; ${ }^{7}$ Instituut voor Sterrenkunde, Katholieke Universiteit Leuven, B-3001 Leuven, Belgium; ${ }^{8}$ Astrophysical Sciences Dept., Princeton University, Princeton, New Jersey 08544 , USA $;^{9}$ Observatoire de Genève, Chemin des Maillettes 51, CH-1290 Sauverny, Switzerland; ${ }^{10}$ Laboratoire d'Astrophysique, Observatoire de Grenoble, Université Joseph Fourier, B.P. 53, F-38041, Grenoble Cedex 9, France; ${ }^{11}$ School of Physics \& Astronomy, University of St Andrews, North Haugh, St Andrews, FIFE, KY16 9SS, United Kingdom; ${ }^{12} 475 \mathrm{~N}$. Charter Street, 5534 Sterling Hall, Madison, WI 53706-1582, USA; ${ }^{13}$ Department of Astronomy, University of Cape Town, Rondebosch 7700, South Africa

Abstract. We report the discovery of both intermediate-order gravity mode and low-order pressure mode pulsation in the same star, HD 209295. It is therefore both a $\gamma$ Doradus and a $\delta$ Scuti star, which makes it the first confirmed member of two classes of pulsating star.

This object is located in a close binary system with an unknown, but likely degenerate companion in an eccentric orbit, and some of the $\gamma$ Doradus pulsation frequencies are exact integer multiples of the orbital frequency. We suggest that these pulsations are tidally excited. HD 209295 may be the progenitor of an intermediate-mass X-Ray binary.

\section{Introduction}

The variability of HD 209295 was discovered by the HIPPARCOS mission (ESA 1997). Handler (1999) performed a frequency analysis of these data and found multiperiodic variability only explicable by $\gamma$ Doradus-type pulsation. As this object is also located in the $\delta$ Scuti instability strip, Handler \& Shobbrook (2002) examined it for short-term variability and indeed discovered short-period pulsations superposed on the $\gamma$ Doradus light curve. 


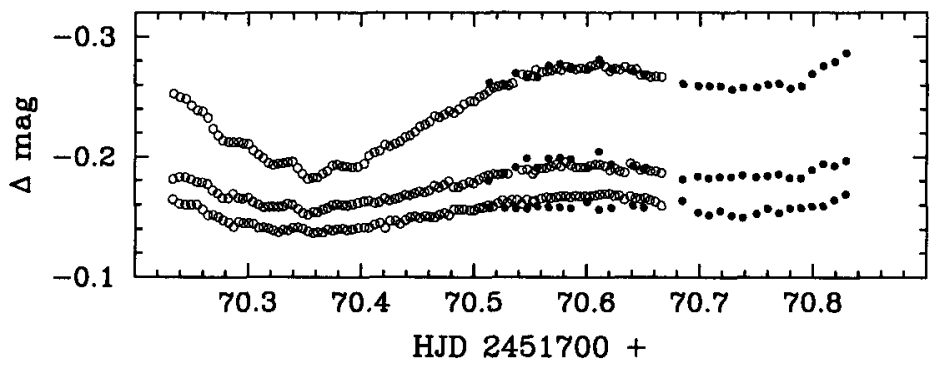

Figure 1. An example light curve of the $\gamma$ Doradus $/ \delta$ Scuti star HD 209295. The open circles are measurements from SAAO and the filled circles are LNA (Brazil) data. The upper light curves in each panel are the $V$ data, the middle ones the $\left(V-I_{c}\right)$ variations and the lowest are the $(B-V)$ light curves. Note the multiperiodic slow and rapid light variability and the corresponding colour changes.

It was then clear that HD 209295 was the first confirmed member of two classes of pulsating star. Consequently, follow-up observations, including a multi-site campaign were organized, resulting in $280 \mathrm{~h}$ of photometric and 135 $\mathrm{h}$ of spectroscopic monitoring. We show an example light curve in Fig. 1.

\section{Results}

The analysis of our measurements yielded several surprises. Firstly, it turned out that the star shows quite large radial velocity variations, in excess of $100 \mathrm{~km} \mathrm{~s}^{-1}$. This was reconciled with binary motion: HD 209295 is a single-lined spectroscopic binary with an orbital period of $3.10575 \mathrm{~d}$ and a rather high eccentricity of 0.35 . The absence of ellipsoidal variability results in an upper limit of the orbital inclination of $44^{\circ}$, which in turn requires a companion mass $>0.98 M_{\odot}$.

We searched for the binary companion which should make itself obvious through an infrared excess if it was a main-sequence star, but could not detect it. However, we noticed that TD-1 satellite measurements of HD 209295 showed quite a large ultraviolet excess, but no binary model with a hot companion subluminous in the optical can explain the amount of the excess flux. The high orbital eccentricity and our constraint on the secondary mass suggest a neutron star as the orbital companion, but this explanation also has its weaknesses, as the star seems chemically normal, has normal space motions, and is not an $\mathrm{X}$-Ray source.

Turning back to the pulsations, we noted little correspondence between the frequency analysis of the photometric and radial velocity measurements. This is at first sight also surprising, but can be explained by the low amplitude of the $\delta$ Scuti pulsations and toroidal corrections due to stellar rotation (Aerts \& Krisciunas, 1996) for the $\gamma$ Doradus modes.

We were able to obtain a mode identification for the two highest-amplitude photometric modes which are weakly present in the line-profile variations by using the method by Telting \& Schrijvers (1997). According to this result, 
both are $\ell=1,|m|=1$. As we have quite a substantial amount of timeseries colour photometry of HD 209295 at our disposal, we also attempted mode identifications following Balona \& Evers (1999) and Koen et al. (1999). However, these results were (at best) inconclusive.

\section{Evidence for forced oscillations}

Another intriguing result from our study was that several of the photometrically detected variations have frequencies which are exact integer multiples of the orbital frequency (see Table 1). This raises the suspicion that these are tidally excited modes.

Table 1. The final multifrequency solution for HD 209295 from multisite photometry from the year 2000. Pulsational phases for zero amplitude are given with respect to one time of periastron passage, HJD 2451771.864 .

\begin{tabular}{|c|c|c|c|c|c|}
\hline ID & Combination & $\begin{array}{c}\text { Frequency } \\
\left(d^{-1}\right)\end{array}$ & $S / N$ & $\begin{array}{c}V \text { Ampl. } \\
(\mathrm{mmag}) \\
\pm 0.2\end{array}$ & $\begin{array}{c}V \text { phase } \\
\left({ }^{\circ}\right)\end{array}$ \\
\hline$f_{1}$ & & $1.12934 \pm 0.00005$ & 63.7 & $38 . \overline{9}$ & $-40.3 \pm 0.3$ \\
\hline$f_{2}$ & & $2.30217 \pm 0.00006$ & 49.9 & 28.7 & $-116.4 \pm 0.4$ \\
\hline$f_{3}$ & $8 f_{\text {orb }}$ & $2.57593 \pm 0.00011$ & 23.2 & 13.2 & $67.2 \pm 0.9$ \\
\hline$f_{4}$ & $f_{2}-f_{1}$ & $1.17283 \pm 0.00004$ & 14.4 & 7.6 & $-73.3 \pm 1.5$ \\
\hline$f_{5}$ & $9 f_{\text {orb }}-f_{1}$ & $1.76859 \pm 0.00005$ & 13.6 & 8.2 & $95.4 \pm 1.4$ \\
\hline$f_{6}$ & $7 f_{\text {orb }}$ & $2.25394 \pm 0.00011$ & 10.6 & 6.6 & $-1.0 \pm 1.8$ \\
\hline$f_{7}$ & $3 f_{\text {orb }}$ & $0.96597 \pm 0.00011$ & 8.9 & 6.2 & $-35.9 \pm 1.9$ \\
\hline$f_{8}$ & $5 f_{\text {orb }}$ & $1.60996 \pm 0.00011$ & 5.8 & 3.9 & $-158.6 \pm 3.0$ \\
\hline$f_{9}$ & $9 f_{\text {orb }}$ & $2.89792 \pm 0.00011$ & 5.7 & 3.5 & $48.9 \pm 3.3$ \\
\hline$f_{A}$ & & $25.9577 \pm 0.0015$ & 5.2 & 1.4 & $162.8 \pm 8.3$ \\
\hline$f_{B}$ & & $13.6873 \pm 0.0018$ & 4.2 & 1.2 & $-3.4 \pm 9.9$ \\
\hline & $f_{\text {orb }}$ & $0.32199 \pm 0.00011$ & & & \\
\hline
\end{tabular}

We note that the photometric colour amplitude ratios of the variations at orbital harmonic frequencies are consistent with those of pulsations. Table 1 also shows that these variations are similarly aligned near periastron (allowing for a $180^{\circ}$ phase ambiguity) which is explained in this scenario as the dominating components of tidally induced $\ell=2,|m|=2$ oscillations (e.g. see Kosovichev \& Severnyj, 1983).

Consequently, one of us (BW) is currently investigating the case for tidally excited oscillations of HD 209295 theoretically, with initially quite encouraging results. We refer to Willems \& Aerts (these proceedings) and Kumar et al. (1995) for more information on theoretical studies of tidal excitation of nonradial oscillations. 


\section{Conclusions}

We showed that HD 209295 is not only the first member of two classes of pulsating star (it is both a $\gamma$ Doradus and a $\delta$ Scuti star), but we have also discovered good evidence for the presence of tidally excited pulsation modes, whereby the responsible binary companion is possibly a neutron star.

The confrontation of photometric and spectroscopic mode identification methods resulted in a reasonable identification for the two dominating $\gamma$ Doradus modes of the star, whereas the photometric techniques did not yield meaningful results. The analysis of the suspected tidally excited modes may give us further insight into this problem.

Despite the many fascinating results obtained from the study of HD 209295, several open questions still remain. We do not properly understand why there is little correspondence between the photometric and radial velocity frequency analyses. The nature of the companion of HD 209295 is also a mystery at present.

Future observational studies of the star, which would be worthwhile, would be an even larger multisite photometric and spectroscopic campaign, in particular with larger telescopes for high-resolution spectroscopy. There are still further pulsational signals to be found. UV and infrared spectroscopy would help to find the orbital companion, and an abundance analysis may reveal an unusual chemical composition caused by the previous evolution of the star through, e.g. a mass transfer stage.

\section{References}

Aerts, C. \& Krisciunas, K. 1996, MNRAS, 278, 877

Balona, L.A. \& Evers, E.A. 1999, MNRAS, 302, 349

ESA, 1997, The Hipparcos and Tycho catalogues, (ESA SP-1200)

Handler, G. 1999, MNRAS, 309, L19

Handler, G. \& Shobbrook, R. R. 2002, MNRAS, in preparation

Koen, C., Van Rooyen, R., Van Wyk, F., \& Marang, F. 1999, MNRAS, 309, 1051

Kumar, P., Ao, C.O., \& Quataert, E.J. 1995, ApJ, 449, 294

Kosovichev, A.G. \& Severnyj, A.B. 1983, Pis'ma v Astronomicheskii Zhurnal, 9,424

Telting, J.H. \& Schrijvers, C. 1997, A\&A, 317, 723 